\title{
La praxis como búsqueda ética en los escenarios de las prácticas formativas $\in$ investigativas*
}

\section{The praxis as ethical search in scenarios of research and training practices}

\section{A prática da pesquisa como cenários éticos $\epsilon$ práticas de formação em investigação}

\author{
Edison Villa Holguín ${ }^{\star *}$ \\ Universidad de San Buenaventura, Medellín, Colombia
}

Resumen. En el presente documento se construyeron algunos elementos de reflexión crítica, a partir de la interpretación de las intenciones, incertidumbres, cuestionamientos, hallazgos y retos que han emergido en el trayecto de acompañamiento sobre las prácticas pedagógicas profesionales, construidas estas, en el entorno de la investigación for-

* $\quad$ Artículo de reflexión resultado del proceso de investigación para optar por el al título de magíster, realizado entre los años 2011 y 2012 en el programa de Maestría en Educación de la Universidad de Medellín. El título del trabajo de grado fue: La praxis como búsqueda ética en el desarrollo de prácticas formativas e investigativas"; proceso de investigación formativa desarrollado en el marco del acompañamiento por parte del Autor a algunas prácticas profesionales y trabajos de grado en la licenciatura con énfasis en Humanidades, Lengua castellana de la Facultad educación de la Universidad de Antioquia, entre los años 2007 a 2010.

** Docente Investigador. Asesor externo. Línea de trabajo derecho humano, formación y conflicto. Grupo interdisciplinario para el desarrollo del pensamiento y la acción dialógica. GIDPAD. Universidad de San Buenaventura, Medellín, Colombia. E-mail: interandinos1@gmail.com y edisonvilla89@hotmail.com 
mativa en el campo de la formación de maestros, a través del método de la sistematización de experiencias, y desde allí se postulan algunas alternativas de construcción ética de los saber para la transformación de la subjetividad del docente de humanidades, visualizando y problematizando las relaciones de dominación que han desbordado históricamente los escenarios de la pedagogía y la interacción discursiva.

Palabras claves. Proyecto ético, formación de maestros, praxis y conflictividad, construcción de subjetividad crítica (Tesauro Unesco).

\begin{abstract}
The current work presents some elements of critical reflection, based on an interpretation of intentions, uncertainties, questioning, findings and challenges resulting in the process of advising professional pedagogical practices. These were carried out in the context of a teacher education research process through a methodological framework of systematization of experiences from which some alternatives of ethical knowledge construction are proposed, aiming at subjectivity transformation processes of humanities pre-service teachers. This work problematizes the relations of domination that have overcome historically pedagogy settings as well as discursive interaction.
\end{abstract}

Keywords. Ethical project, teacher education, praxis and conflict, critical subjectivity construction (Thesaurus Unesco).

Resumo. Neste trabalho nós construímos alguns elementos de reflexão crítica a partir da interpretação das intenções, incertezas, dúvidas, descobertas e desafios que surgiram no caminho de acompanhamento sobre as práticas de ensino profissional construída estes, nas proximidades do pesquisa formativa na área de formação de professores, através do método de sistematização de experiências, ea partir daí você postular alguma construção ética alternativa de conhecimento para a transformação da subjetividade humanidades professor, visualizar e problematizar as relações de dominação que historicamente inundado cenários pedagogia e interação discursiva.

Palavras chave. Projeto ético, formação de professores, a prática e os conflitos, construir a subjetividade crítica (Tesauro Unesco). 


\section{El ejercicio de la praxis formativa y su aporte en la construcción de un proyecto ético}

En el contexto de cambio de la era informacional con el que arranca el siglo XXI, se han tratado y propuesto masivamente, dos dimensiones de trabajo para la mediación con el conocimiento, como lo son: la epistémica de flujos y registros de datos e información y la asunción de las políticas de la comunicación y difusión eficaces para el proceder con los aprendices. Pero ¿Cuál es ese marco ético en el que se hace hoy la construcción subjetiva e intersubjetiva del conocimiento y la realidad? Parece que ya no son las religiones y menos los partidos políticos los que lo determinan, todo indica que es la ley del comercio y sus mediaciones espectáculo, las que inauguran unas nuevas formas "didácticas" y unos nuevos pactos relacionales "educativos".

El presente proceso con pretensiones de formación ética, enfatiza en la necesidad de visualizar y potenciar dentro de dichas estructuras de superficie, un elemento de fondo, que nos libere del pragmatismo absoluto, de solo ser fichas funcionales a las condiciones en las que están andando los proyectos culturalmente hegemonizados en la pedagogía. Se entiende desde este ejercicio reflexivo, que la tarea histórica que hoy ronda la pedagogía, tiene que ver con la necesidad de potenciar los espacios y las acciones formativas e investigativas acordes a las transformaciones sociales en que los proyectos culturales de cambio vienen desarrollándose en nuestra contemporaneidad.

Con conocimiento de causa sobre el espacio reducido que tiene la proyección ética en los maestros de humanidades, como sujetos formadores de otros sujetos que buscan pensarse en términos de cómo construir sus posiciones como maestros frente a lo que les rodea, desde la toma de decisiones con autonomía, en el trámite de sus propias contradicciones y las que el medio les condiciona, se busca visualizar desde este ensayo, ideas que posibiliten construir una ética del proceso educativo que se consolide con praxis, pensamiento crítico y dialogicidad.

Imbernon (1999) cita autores como Flecha, Sacristán, Giroux, Mc Laren y otros que muestran las búsquedas del sujeto docente, tratando de entender el modelo de sociedad presente, ya sea para acomodarse a él, confrontándolo y exigiendo ser incluidos, o resistiéndolo, construyéndole un paralelo. Configurándose así tendencias y actitudes algunas más 
pragmáticas, otras más reflexivas en la búsqueda por ser funcionales o constructores de horizontes dentro del que hacer del campo docente. Aportes que concluyen postulando la necesidad de entender el contexto contemporáneo, que ya no resiste el capricho de seguir congelando la realidad del acceso al conocimiento en las rejas y los muros de las escuelas clásicas.

Bruner (1996) nos deja algunos postulados de los cuales se infiere que la construcción de realidad por parte del sujeto, se hace no solo en el esquema mental individual, sino en el proceso relacional con los otros, Estas relaciones a su vez son constituidas por unos mitos de explicación, rituales, pactos y acuerdos que nos pre-existen pero que a la vez también se re-editan con cada época y cada contexto. Los conocimientos y las realidades que tenemos en la contemporaneidad son construcciones sociales elaboradas en un marco valorico-axiológico a descifrar, desde donde emergen las éticas de relación y vinculo de lo común y lo público.

Bruner (1996) argumenta que "la realidad" que atribuimos a los "mundos" que habitamos es construida. Se hace, no se encuentra; en este sentido, la educación ayuda a construir el fondo ético relacional para que los sujetos aprendan a usar las herramientas de creación de significado y concepción de la realidad, como el producto de la creación de conocimiento conformada a lo largo del contacto con los acumulados "caja de herramientas de formas de pensar de una cultura". Para adaptarse comprensivamente al mundo en el que nos encontramos y para participar en el proceso de cambiarlo según el requerimiento de un tipo de sociedad. "somos la especie intersubjetiva por excelencia. Es esto lo que nos permite "negociar" los significados cuando las palabras pierden el mundo" (p. 39).

Gracias al constructivismo la tradición pedagógica occidental empieza a considerar la importancia de la ínter-subjetividad, no solo en el modelo clásico de la transmisión cultural, sino como el sujeto participante de la construcción de la misma. En este contexto, la educación pensada como una ética de lo público y lo común, saber cultural que nos recoge y asume como sujetos, hace que el aula se re-conceptualice como comunidad de aprendices mutuos. En la educación la ética es el vínculo relacional que hace común- unidad, pues las comunidades de aprendices mutuos producen obras colectivas y sostienen la solidaridad grupal o zona de desarrollo próximo como lo pensaba Vigotsky (1988): 
...la distancia en el nivel real de desarrollo, determinado por la capacidad de resolver independientemente un problema, y el nivel de desarrollo potencial, determinado a través de la resolución de un problema bajo la guía de un adulto o en colaboración con otro compañero más capaz (p. 133).

Sin embargo, la discusión central de una posible proyección ética, apunta a la necesidad de evaluar los aspectos políticos y éticos que tanto se han promulgado a través de cuestionar el tipo de relaciones resultantes de los procesos educativos; en vista de que el sentido y el criterio para evaluar el éxito educativo tiene que ver con el tipo de bienestar en el que vive una comunidad. ¿Qué tipo de relaciones están siendo producidas educativamente, por quienes y por qué? Se nos hace necesario indagar a este respecto, sobre todo porque desde la educación debe ser problematizada la calidad de las relaciones que se crean en la sociedad.

Paulo Freire (2009) tenía preguntas parecidas y su búsqueda de siempre fue la construcción de una ética dialógica para una mejor sociedad, que debía darse por medio de la educación y la comunicación. Su trayectoria pedagógica marco la mayoría de los discursos transformadores de finales de siglo XX, de allí que sus lectores y simpatizantes queramos asumirnos como sujetos constructores de otra historia educativa, sujetos de la búsqueda, de la pregunta, de la decisión, de la ruptura, de la opción, sujetos éticos.

En palabras de Freire (2009):

La ética de que hablo no es una ética menor, restrictiva, del mercado, que se inclina obediente a los intereses del lucro. En el nivel internacional comienza a aparecer una tendencia a aceptar los reflejos cruciales del "nuevo orden mundial" como naturales e inevitables. (...) No hablo, obviamente, de esta ética. Hablo, por el contrario, de la ética universal del ser humano. De la ética que condena el cinismo del discurso arriba citado que condena la explotación de la fuerza de trabajo del ser humano, que condena acusar para oír decir, afirmar que alguien dijo A sabiendo que dijo B, falsear la verdad, engañar al incauto, golpear al débil y al indefenso, sepultar el sueño y la utopía, prometer sabiendo que no se cumplirá la promesa, testimoniar mentirosamente, hablar mal de los otros por el gusto de hablar mal. La ética de que hablo es la que se sabe traicionada y negada 
en los comportamientos groseramente inmorales como la perversión hipócrita de la pureza en puritanismo. La ética de que hablo es la que se sabe afrontada por la manifestación discriminatoria de raza, género, clase. Es por esa ética inseparable de la práctica educativa, no importa si trabajamos con niños, jóvenes o adultos, por la que debemos luchar. Y la mejor manera de luchar por ella es vivirla en nuestra práctica, testimoniarla con energía, a los educandos en nuestras relaciones con ellos (pp. 17-18).

Partiendo de las ideas Freirianas, una propuesta ética es insuficiente al considerar solamente una manera de mirar y comprender el mundo. Pues no se trata de pensar la ética como la construcción utópica única que armoniza o la idea de comunidad basada en la unión y la cordialidad inmanente. Ni se trata de plantear que las relaciones educativas dialógicas son automáticamente democráticas, en busca del absolutismo y la uniformidad. Para Laclau (1998, p. 93) en una sociedad democrática existe el debate y la discusión, y las relaciones de conflicto son sostenidas, no eliminadas. Desde estos puntos de vista, ser ético implica asumir y darle importancia a las tensiones y conflictos usuales en las sociedades. Democracia no es igual a consenso, es aprender a vivir racionalmente con el disenso.

La comprensión de la variedad de mundos posibles no se lleva a cabo desde una posición única o macartista, en tanto en los proyectos pedagógicos, está en juego la construcción constante de un horizonte crítico, que permita comprender las teorías pedagógicas, la historia y la epistemología de las ciencias del lenguaje, los estudios literarios y la pedagogía, las tendencias educativas, los contextos y los saberes socioculturales con el propósito de "examinar, desmantelar, analizar, poner entre paréntesis, destruir y reconstruir las prácticas pedagógicas" (McLaren, 1997, p. 55), y de este modo generar condiciones para que los/ as maestro/as elaboren lecturas críticas de sí mismos/as y del mundo. De este planteamiento de McLaren (1997) se desprenden preguntas por las condiciones de producción del significado, y en términos generales por las relaciones entre el lenguaje, la enseñanza, la formación y el poder en el aula y la vida escolar, pero también en otros campos abiertos por la reflexión sobre los medios de comunicación, la publicidad y el arte.

Una posición ética implicara entender que si la realidad se construye socialmente, hay diversidad de construcciones nucleadas por lógicas 
diferentes que responden a condiciones culturales, sociales, históricas, generacionales y políticas diferentes, al corresponder a diferentes universos simbólicos, cuando interactúan generan fricción y tensión. La tensión entendida no como oposición excluyente, sino como relación de fuerzas, las tensiones develan que las diferentes lógicas responden también a relaciones de poder; muestran, en últimas la íntima relación entre lo lógico, lo ético y lo estético.

Cuando nos referimos a las tensiones no lo hacemos desde una óptica que las presente como obstáculos para la interacción, sino todo lo contrario, la aparición de una tensión es señal de que las relaciones de poder se movilizan y sufren transformaciones, señal de que hay resistencia. Dice Garavito (1999):

Cada día se forman nuevos hábitos, pero también cada día participamos en nuevos actos de resistencia y de creatividad, así sea una manera de jugar, de hablar o de caminar. Hay un pesimismo mórbido al decir que los poderes impedirán la creatividad. Ningún poder, por despótico que sea podrá jamás impedir la creatividad minoritaria (p. 316).

Así, mientras haya relaciones de poder, habrá resistencias; en este sentido, Foucault (1993) dice que éstas:

... son más reales y eficaces cuando se forman allí mismo donde se ejercen las relaciones de poder; la resistencia al poder no tiene que venir de afuera para ser real, pero tampoco está atrapada por ser la compatriota del poder. Existe porque está allí donde el poder está: es pues como él, múltiple e integrable en estrategias globales (p. 171).

De esta forma, el proceso presente asume una búsqueda investigativa en la que la ética apunta críticamente hacia la configuración de formas de resistencia de maestras y maestro/as frente a la imagen de sujetos subalternos (en relación con la ciencia, el estado y otras entidades). En esta vía, el estudio de las relaciones entre subjetividad y contexto se enmarca en el interrogante por el sentido que tiene para maestras y maestro/as el investigar, pero, ¿̇a qué llamamos investigar?, ¿qué relaciones de saber-poder están implicadas? y żqué tiene ello que ver con la identidad narrativa de los sujetos y la comunidad de un programa en el que literatura y lenguaje constituyen dos grandes intereses? 
Éstas y otras preguntas surgen en un proceso que teje y desteje relatos a punta de múltiples experiencias, miradas y voces, y en los que lenguaje narrativo y referencial, al igual que en la vida cotidiana, se encuentran en una trama que desde el primer momento propone una forma de comprensión investigativa diferente que no persigue la generalización sino la "transferibilidad" (Connelly y Clandinin, 1995, p. 31), por vía analógica.

El interrogante central del cual se parte tiene que ver con ¿Cuál es el proyecto ético que está en el trasfondo de las prácticas investigativas y formativas de las humanidades? Asunto que no se resuelve con los debates sobre el sujeto en formación. Sino que además exige que el sujeto de la educación en humanidades indague y comprenda el contexto histórico y filosófico contemporáneo, como una manera de empezar a tramitar la relación dialéctica entre conflicto y educación y desde allí constituir diversas propuestas investigativas y formativas, donde se visualicen y problematicen los factores contextuales que generan situaciones problemicas en lo especial del campo educativo y que han condicionado la manera en que la academia contemporánea entiende y realiza prácticas sociales como la educación y la comunicación operadas por fuera de un marco de ética y responsabilidad con el humanismo.

\section{¿Cómo entender el concepto "praxis"?}

El panorama formativo de las humanidades en el campo pedagógico por las dinámicas y las circunstancias donde estas se dan hoy, es impensable sin las tensiones profesionales entre corrientes como el academicismo, el pragmatismo y el contextualismo, que exigen el presente tipo de ejercicios de problematización, derivándose una serie de preguntas que se buscó tramitarlas desde el ejercicio de praxis. Antes de detallar el proceso vivido, es importante dejar claro para el desarrollo de esta propuesta que se ha entendido por praxis.

La educación está inscrita en un medio complejo, sujeta a relaciones y tensiones que confrontan desiguales modos de encarar la producción, las ideologías y los sistemas de poder. Se ha observado a lo largo de este proceso, como el pragmatismo funcional y aplicado desde las dinámicas sociales de la educación, en su mayoría, no han posibilitado a los procesos de prácticas ser acompañadas de la reflexión y discusión epistemológica propuesta en la perspectiva de la praxis, lo cual influye para que la fundamentación teórica, técnica y formativa del docente en 
formación, no delimite, ni elabore un distanciamiento ideológico que le permita tomar decisiones en términos de los abordajes y los enfoques que condicionan la funcionalidad de su hacer profesional.

En particular, se puede por medio del ejercicio de praxis nombrar de forma testimonial las vividas en la Facultad de Educación de la Universidad de Antioquia entre los años 2007- 2010 a partir de las experiencias acontecidas en el programa de humanidades, en el trayecto de acompañamiento a los grupos de prácticas profesionales y trabajos de grado, que han sido el epicentro del presente proceso investigativo. Al hablar de la necesidad de que los docentes en formación se constituyan y consoliden como sujetos políticos, constructores de sus propios desarrollos en el ámbito educativo, artífices de sus destinos disciplinares como humanistas, con herramientas críticas, teóricas y metodológicas para incidir en la realidad profesional que les circunda, nos insertamos en la concepción del sujeto como el sujeto de la praxis, es decir el sujeto que se transforma a sí mismo y a su entorno.

La praxis, desde la perspectiva propuesta, posibilita que se exprese reflexivamente lo que la práctica oculta, y promueve acciones que fomentan la reflexividad y la toma de conciencia, a partir de la problematización y la proposición de alternativas de resistencia a los entramados ideológicos de nuestro contexto, generando y facilitando los ambientes para que emerjan actitudes activas, críticas y participativas desde nuestro que hacer como educadores, buscando la transformación de las estructuras que se reflejan en las prácticas socio- históricas de la educación como lo son la homogenización cultural, el autoritarismo y el absolutismo cientificista.

"Praxis" es una palabra griega que desde los postulados aristotélicos se asocia con la noción de "practica" o acción concreta. Las múltiples interpretaciones sobre el concepto involucran relaciones dicotómicas entre teoría y práctica, dichas relaciones se han elaborado desde diversas perspectivas: valor práctico, teoría para la acción y equilibrio del binario acción- reflexión. Para el caso de la socio-critica, como enfoque que se comparte desde la presente propuesta, se asume la perspectiva de la complementariedad en los términos: praxis como unidad dialéctica y acción teorizada. Este desarrollo histórico del concepto nos ha ido llevado a asumir acción y el pensamiento en una misma categoría. Entendiéndola como una acción que tiene efecto en sí misma, buscando su cualificación, pensando sobre sí y sobre lo que se hace. 
El análisis marxista de las prácticas humanas ha profundizado de manera importante en el concepto proponiéndolo como: práctica revolucionaria o transformadora que busca la superación del sentido común o inmediato que se adopta en la conciencia ordinaria, cotidianidad que vive sin razón crítica, sumida al ámbito práctico, condicionado por el accionar naturalizado o normalizado al cuál se propone se enfrente con pensamientos críticos.

Para Paulo Freire (1984, p. 79) indica que la praxis, la reflexión y la acción son indisolubles; por tanto no es posible negar la relación que se constituye entre saber y acción. La negación de uno de los elementos del par desvirtúa la praxis, transformándola en activismo o un subjetivismo, siendo cualquiera de los dos una forma errónea de captar la realidad.

Por ello, contrario a los enfoques de la exclusividad de la acción concreta, promocionados como pragmatismo y que reducen la praxis al valor práctico para satisfacer necesidades o a la razón utilitaria que entiende lo productivo solo en lo práctico, los pensamientos críticos se valen de la visibilización y problematización del hecho de que el humano que vive del sentido común, buscando resolver los problemas de una manera práctica, termina agudizándolos a partir de lo inmerso que se encuentra en el entramado ideológico del orden social que lo predetermina, por lo general en función de intereses y beneficiarios ajenos.

Desde la praxis como herramienta de análisis, las corrientes socio- criticas proponen otra manera de tramitar las contradicciones que se generan al vivir dentro de los entramados ideológicos, concientizando y problematizando las prácticas y las relaciones sociales y humanas buscando la transformación de dichas acciones en función de un proceso de experiencia critica desde las subjetividades y las colectividades en las que nos encontramos.

En el ámbito educativo que circunda esta experiencia investigación participante se trataría de trabajar de forma procesual con los grupos y comunidades educativas para que se elaboren análisis de los contextos situacionales buscando integrar interpretaciones de las realidades desde múltiples variables, caracterizando escenarios, tiempos, actores sociales, relaciones de poder, y expresiones desde la diversidad de códigos que nos posibiliten descubrir mensajes ocultos y vincular acontecimientos sociales, macros y micros, como formas de acercarnos a la problematización de los roles y prácticas impuestas y naturalizadas del mundo escolar. Para después a partir de una devolución sistemática de dicha elaboración de 
conocimientos, establecer alternativas de acción y decisión que desencadene en prácticas cualificadas de acuerdo a una intención, fenómeno que desde los procesos formativos se nombra como transformación.

Las pistas metodológicas anteriormente nombradas nos exigirán avanzar en el desarrollo procesual de la praxis formativa que se construye en los tipos y estilos de preguntas, en la caracterización de fórmulas y modelos, prescripciones y desarrollos llevados a cabo en el mundo educativo occidental. Esta experiencia nos posibilita a los educadores entender los supuestos de los diferentes paradigmas en los que vive la comunidad educativa, y que implica nos reconozcamos en los referentes y modelos instaurados en las intervenciones que llevamos a cabo con nuestros acompañamientos y discursos y en ellos el desvelamiento de sus contenidos, intereses, finalidades, acciones y formas de entender al otro y a lo otro, elementos que se nos convierten en claves de lectura, comprensión y proposición a la hora de construir una perspectiva educativa alternativa, que rompa con los proyectos instrumentalizadores de los sujetos y los procesos.

Lo vivido en el proceso nos permite afirmar que la filosofía de la praxis, además de que favorece la lectura de la realidad contextual en clave de las relaciones de dominación, como condicionante de la dinámica educativa, posibilita la apropiación crítica y auto-critica de las propias prácticas éticas y epistémicas, cualificando el acompañamiento a los grupos desde un pensamiento diverso, mixto y de transformación permanente. Como lo indica Gramsci (2000):

Una filosofía de la praxis no puede dejar de presentarse inicialmente con una actitud polémica y crítica, como superación del modo de pensar precedente y del pensamiento concreto existente (o del mundo cultural existente). Es decir, debe presentarse ante todo como crítica del sentido común (p. 9).

\section{Caracterización de un posible proyecto ético de acción transformadora}

Esta experiencia reflexiva asumió un formato dialéctico y a partir de una perspectiva de comprensión, construyo un texto en la tensión de reflejar el dialogo entre diferentes voces y diversas expectativas que se escuchan en el terreno educativo escogido para el proceso de estudio investigativo, es decir la formación docente, asumiendo el costo de un 
proceso formativo de largo alcance, donde los impactos inmediatos están, pero no se dejan ver a la hora de pensar en las transformaciones educativas de fondo.

Los ejercicios de comprensión e interpretación en formato crítico de la realidad para darse cuenta del propio proceso de opresión por el que atraviesa cada sujeto y la posterior problematización o concientización como diría Freire (2005, p. 53), desde el auto aprendizaje, la construcción de subjetividad y la producción colectiva de sentidos y significados, estuvieron en función de generar un proyecto educativo comunitario, es decir, se pensó en la realidad organizativa de la educación desde el docente hacia una mejor sociedad, que se va construyendo en nosotros cuando cualificamos las relaciones humanas, objeto y fin último del campo formativo, para vivir de otras maneras los conflictos y tramitar con humanismo los intereses que emergen de la relación entre saber y poder.

En el análisis colectivo de los registros escritos, se evidenciaron: las configuraciones didácticas decadentes que por su esencia bancaria y enciclopédica condicionan la posibilidad real de intercambio de saberes y la construcción de aprendizajes Además se identifica una dimensión política arrebatada al sujeto educativo, por los dispositivos de dominación en representación del aparato institucional. Fenómenos que obedecen a trasfondos discursivos o guiones culturales preestablecidos conservados para la cosificación y la funcionalidad acrítica.

Desde allí se advierte la urgente necesidad de reconstruir o resignificar las practicas, en otros fines, resaltando la importancia de la autonomía docente para tomar decisiones sobre el tipo de práctica formativa que se quiere impulsar, el cómo y sus consecuencias. Implementando acciones de mejoramiento y cualificación a partir de la caracterización de los problemas y los hallazgos que emergieron en el trámite de estos, re-dimensionando las categorías de lo formativo, lo didáctico y lo crítico, como instrumentos que pueden jugar a favor de la transformación de la escuela, reconstruyendo la imagen del maestro desde la figura del intelectual orgánico (Gramsci, p. 2000, p. 5), recuperando su sano protagonismo en el proceso, asumiéndose el docente como el responsable de tejer las relaciones entre la producción teórica y los saberes acumulados en la experiencia de la práctica social de la educación. 
En la búsqueda y en las intenciones del presente proceso nos encontramos con la necesidad y la emergencia de la configuración de otros pactos, otros acuerdos y otros códigos éticos para la vivencia de un proyecto escolar diferente, y este pasa por la definición del propio proyecto ético del maestro desde la filosofía de otro humanismo, que frente a la coyuntura de decadencia por la que pasa la institución escolar y en general la sociedad occidental, lleve a cabo en paralelo a la visión programática del sistema, pero con la intención y el compromiso de ir incidiendo en el funcionamiento hacia el mediano plazo, un proceso pedagógico interdisciplinar que asuma la reflexión crítica como contenido, en el análisis sobre los problemas derivados de las relaciones acaecidas en la profesión, generando la capacidad de transformación en comunidad y desde el medio que le rodea, manteniendo sus clases como escenarios permanentes de trabajo a profundidad, en otros intercambios humanos que se nutran y se conjuguen en lo plural, lo mixto y lo diverso, problematizando, concientizando y transformando en el aquí y en el ahora con sus ejercicios de praxis.

En conclusión, entendemos por praxis la práctica reflexionada sobre nuestras prácticas sociales que articulan procesos de producción de conocimiento, recreación de saberes, reflexión y promoción de acciones para entendernos como sujetos protagonistas de nuestra propia historia formativa. El desarrollo del pensamiento crítico y la comprensión de la realidad de los escenarios de formación investigativa hacia la consolidación de subjetividad docente problematizada, es un asunto de principios éticos, políticos y epistémicos de sujetos que se auto-determinan y deciden realizar las transformaciones necesarias a sus contextos inmediatos, a partir de trascender las tendencias y las actitudes que han puesto énfasis en el reclamo al sistema, priorizando la incidencia en la cualificación de las relaciones intersubjetivas que se propician en los escenarios del intercambio discursivo del campo educativo.

\section{Referencias}

Bruner, J. (1996). La Educación puerta de la cultura. Madrid: Editorial Visor.

Connelly y Clandinin (1995). Relatos de experiencias e investigación narrativa. En Déjame que te cuente. Ensayos sobre narrativa y educación. Barcelona: Laertes.

Freire, P. (1970). Pedagogía del oprimido. Montevideo: Paz y Terra. 
Freire, P. (1984). Educación como práctica de la libertad. México: Siglo XXI Editores.

Foucault, M. (1993). Microfísica del poder. Madrid: Edición La Piqueta.

Freire, P. (2005). Pedagogía del oprimido. México: Siglo XXI Editores.

Freire, P. (2009). Pedagogía de la autonomía. México: Siglo XXI Editores.

Garavito, E. (1999). Escritos escogidos. Medellín: Editorial Universidad Nacional.

Gramsci, A. (2000). Los intelectuales y la organización de la cultura. Buenos Aires: Editorial Nueva Visión.

Imbernon, F. (1999). La Educación en el siglo XXI: los retos del futuro inmediato. Barcelona. Grao.

Laclau, E. (1998). Deconstrucción, Pragmatismo y Hegemonía. En Deconstrucción y Pragmatismo, Chantal Mouffe (Comp.). Buenos Aires: Paidós.

McLaren, P. (1997). Pedagogía crítica y cultura depredadora. Políticas de oposición en la era posmoderna. (Trad. al español por Pilar Pineda Herrero). Barcelona: Ed. Paidós.

Vigotsky, L. (1988). El desarrollo de los procesos psicológicos superiores. México: Editorial Crítica- Grupo editorial Grijalbo. 